\title{
The Synthesis and New Crystal Structures of Three
}

\section{Arylpiperazine Compounds}

\author{
Jinhui Zhou 1,2, Maocai Yan ${ }^{1}$, Maojian Shi ${ }^{1}$, Jun $\mathrm{Xu}^{3}$ and Guoqiang Shangguan 1,* \\ ${ }^{1}$ College of Pharmacy, Jining Medical University, Rizhao 276826, China \\ ${ }^{2}$ School of Chemistry and Chemical Engineering, University of Shandong, Jinan 250100, China \\ ${ }^{3}$ Department of Chemistry, University of Zurich, 190CH-80577 Zurich, Switzerland \\ *Correspondence: gqshangguan@mail.jnmc.edu.cn; Tel.: +86-633-298-3730
}

\begin{abstract}
The structure of arylpiperazine moieties as an important pharmacophore could generate wide pharmacological activities. Arylpiperazine derivatives referred in this paper possessed antitumor activity. The title compounds were crystallized by slow evaporation. 2-(4-(2-(4-Phenylpiperazin-1-yl)ethyl)benzyl)isoindoline-1,3-dione(1),

2-(4-(2-(4-(4-Bromophenyl)piperazin-1-yl)ethyl)benzyl)isoindoline-1,3-dione

(2) and

2-(4-(2-(4-(4-Chlorophenyl)piperazin-1-yl)ethyl)benzyl)isoindoline-1,3-dione (3) crystallizes in the triclinic space group P-1 with two molecules in the unit cell. The unit cell parameters for $\mathbf{1}$ are $\mathrm{a}=$ 6.9159(14) $\AA, \mathrm{b}=9.999(2) \AA, \mathrm{c}=16.925(3) \AA, \alpha=88.25(3)^{\circ}, \beta=85.14(3)^{\circ}, \gamma=79.22(3)^{\circ}$. The unit cell parameters for 2 are $\mathrm{a}=6.9995(14) \AA, \mathrm{b}=9.919(2) \AA, \mathrm{c}=17.671(4) \AA, \alpha=97.55(3)^{\circ}, \beta=92.19(3)^{\circ}, \gamma=$ $102.23(3)^{\circ}$. The unit cell parameters for 1 are $a=6.9872(14) \AA, b=9.863(2) \AA, c=17.557(4) \AA$, $\alpha=96.81(3)^{\circ}, \beta=91.89(3)^{\circ}, \gamma=101.74(3)^{\circ}$. Pi-pi interactions were observed between molecules, and compound 2 and 3 showed halogen interactions between molecules nearby, which was different from compound 1 .
\end{abstract}

Keywords: arylpiperazine compound; X-ray crystal structure; characterization; triclinic

\section{Introduction}

The structure of arylpiperazine moieties as an important pharmacophore could generate wide pharmacological activities [1-3]. Compounds with arylpiperazine moieties have anti-proliferative properties [4-5]. Naftopidil, an arylpiperazine ether derivative, is a specific a1d-adrenergic receptor antagonist [6,7], and it is one of the most widely used a1-adrenergic receptor antagonists in Japan for the treatment of benign prostatic hyperplasia (BPH) [8,9]. Recent studies have shown that naftopidil could possibly exert an anticancer effect and inhibit prostate cancer cell growth by arresting the G1 cell cycle phase [10,11], as well as inducing apoptosis in malignant mesothelioma cell lines [12]. Arylpiperazine derivatives referred in this paper possessed antitumor activity.

The halogen bond is a noncovalent interaction involving polarizable chlorine, bromine, or iodine molecular substituents. It is easily observed in small molecule packing [13,14], and is now being exploited to control the assembly of small molecules in the design of supramolecular complexes and new materials $[15,16]$. In this study, arylpiperazine halogen derivatives were synthesized to observe if the packing mode was affected by halogen bonds.

\section{Results}

\subsection{Description of Structures}

Single-crystal X-ray diffraction reveals that all the three compounds crystallized in the triclinic space group P-1. The molecular structures and unit cell of three compounds are shown in Fig. 1 and Fig.2, respectively. All asymmetrical units contain only one independent molecule. The chemical structures of these three compounds were similar, except the substituent groups at ring 1 
(C1-C2-C3-C4-C5-C6). Compared with compound 1, compound 2 and 3 were substituted by $\mathrm{Cl}$ and $\mathrm{Br}$, respectively. All three compounds contained three benzene rings, a six-membered ring, apyrrole ring. Ring 4 and ring 5 are coplanar. In compound 1, the dihedral angles made by the planes of the ring1 and ring2, ring 1 and ring 3, ring 1 and ring 4are3.9(2) ${ }^{\circ}, 110.5(2)^{\circ}$ and $67.5(2)^{\circ}$, respectively.In compound 2, the dihedral angles made by the planes of the ring 1 and ring 2, ring 1 and ring 3 , ring 1 and ring 4are16.9(2) ${ }^{\circ}, 92.0(1)^{\circ}$ and $101.0(1)^{\circ}$, respectively.In compound 3 , the dihedral angles made by the planes of the ring1 and ring 2, ring 1 and ring 3, ring 1 and ring $4 \operatorname{are} 17.4(2)^{\circ}, 91.4(2)^{\circ}$ and $99.5(2)^{\circ}$, respectively. The bond lengths of these 3 compounds were same, which indicated that substitution of halogen didn't affect the configuration of the molecules. The dihedral angle values indicated the conformation of compound $\mathbf{2}$ and 3 were similar, which was a little different from that of compound $\mathbf{1}$ due to the effect of the halogen atoms.

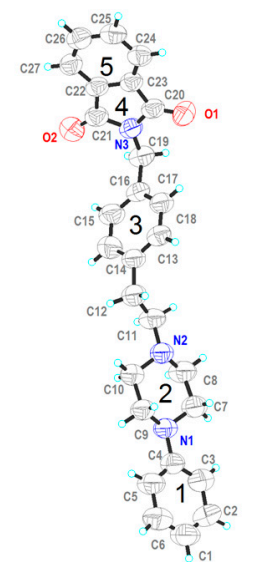

(a)

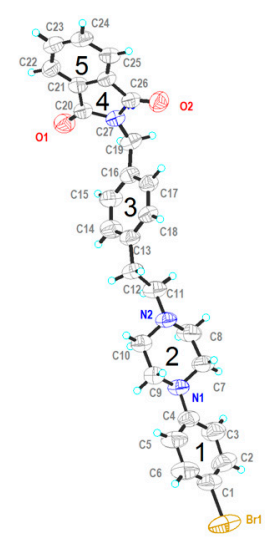

(b)

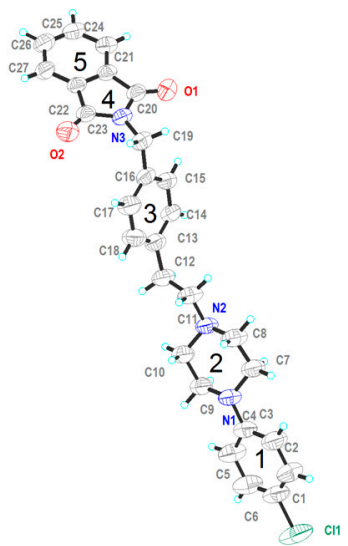

(c)

Figure 1.The molecular structure of compound 1(a), compound 2(b), compound 3(c).Color codes: C (gray), $\mathrm{O}$ (red), $\mathrm{N}$ (blue), $\mathrm{H}$ (green), Br(dark yellow), $\mathrm{Cl}$ (dark green). Displacement ellipsoids are drawn at the $30 \%$ probability level.

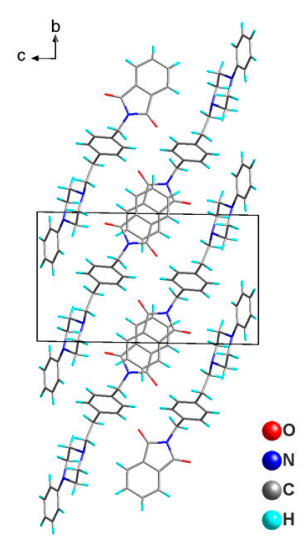

(a)

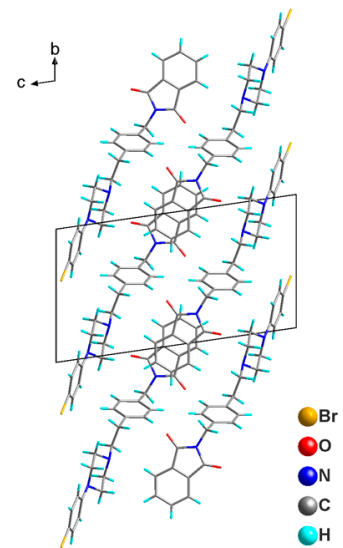

(b)

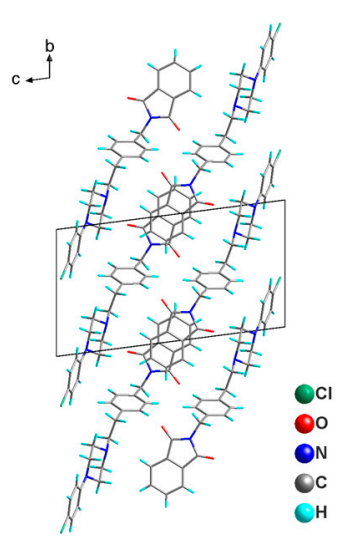

(c)

Figure 2.Unit cells of compound 1(a), compound 2(b), compound 3(c).

The bond lengths and bond angles in Compounds 1, 2 and 3 listed below.

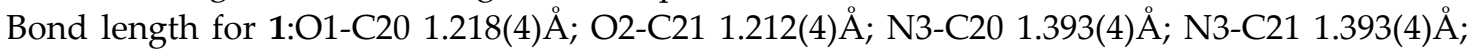

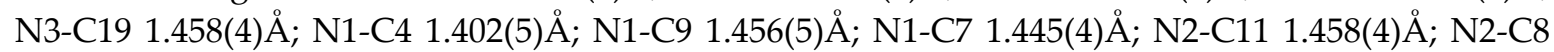

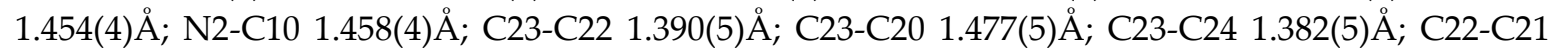

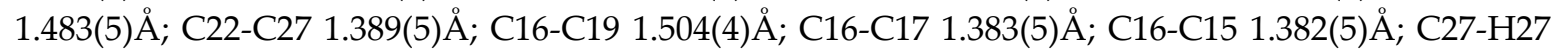

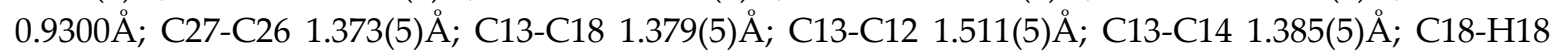

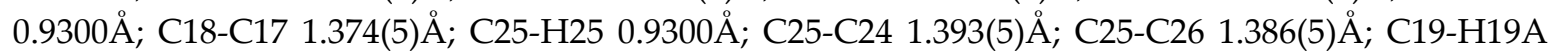

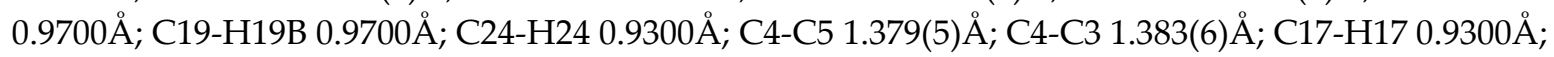




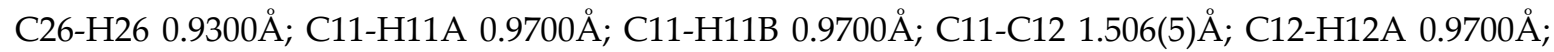

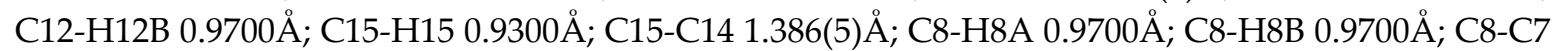

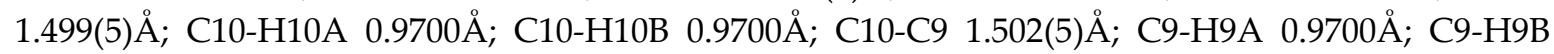

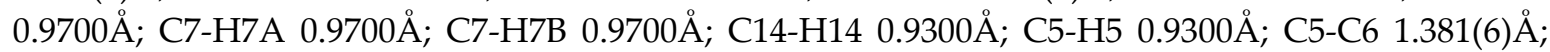

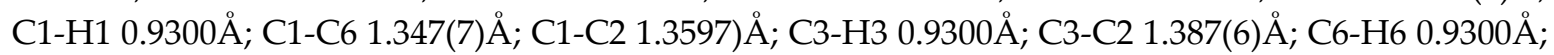
C2-H2 0.9300.

Bond angle for 1: C20-N3-C21 111.6(3) ${ }^{\circ}$; C20-N3-C19 124.7(3) ${ }^{\circ}$; C21-N3-C19 123.6(3) ${ }^{\circ}$ C4-N1-C9 117.8(3) ; C4-N1-C7 117.1(3) ; C7-N1-C9 110.7(3) ${ }^{\circ}$; C11-N2-C10 113.2(3) ${ }^{\circ}$; C8-N2-C11 110.2(3) ; C8-N2-C10 107.5(3) ; C22-C23-C20 107.6(3) ; C24-C23-C22 121.3(3) ; C24-C23-C20 131.1(4) ${ }^{\circ}$; C23-C22-C21 108.3(3) ; C27-C22-C23 120.7(3) ; C27-C22-C21 130.9(4) ; O1-C20-N3 124.3(3) ; O1-C20-C23 129.1(4) ${ }^{\circ} ; \quad \mathrm{N} 3-\mathrm{C} 20-\mathrm{C} 23 \quad 106.6(3)^{\circ} ; \quad \mathrm{O} 2-\mathrm{C} 21-\mathrm{N} 3 \quad 124.9(4)^{\circ} ; \quad \mathrm{O} 2-\mathrm{C} 21-\mathrm{C} 22 \quad 129.2(4)^{\circ}$; N3-C21-C22 105.9(3) ${ }^{\circ}$; C17-C16-C19 120.6(3) ; C15-C16-C19 121.7(3) ; C15-C16-C17 117.7(3) ; C22-C27-H27 121.0 ; C26-C27-C22 118.0(4) ; C26-C27-H27 121.0 ; C18-C13-C12 120.6(4) ${ }^{\circ}$;

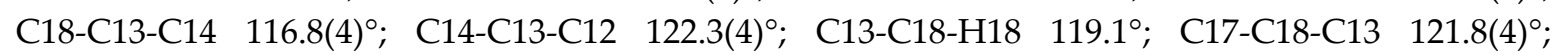
C17-C18-H18 119.1; C24-C25-H25 119.6 ; C26-C25-H25 119.6 ; C26-C25-C24 120.8(4) ${ }^{\circ}$; N3-C19-C16

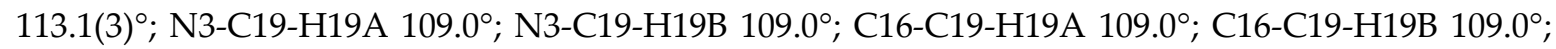
H19A-C19-H19B 107.8 ; C23-C24-C25 117.6(4) ${ }^{\circ}$; C23-C24-H24 121.2 ; C25-C24-H24 121.2 ; C5-C4-N1

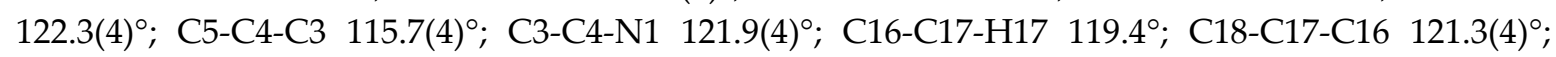

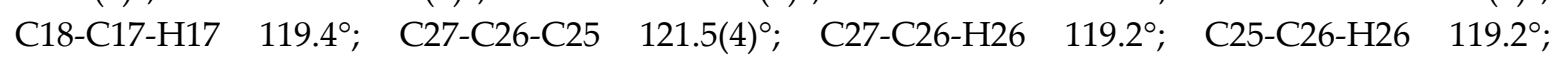

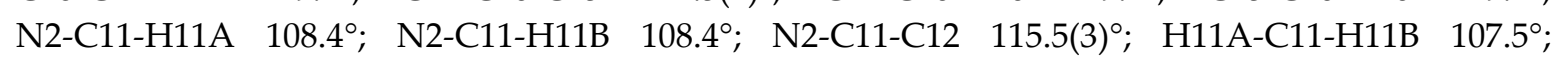

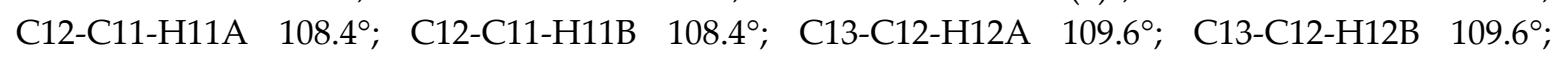
C11-C12-C13 110.3(3) ; C11-C12-H12A 109.6 ; C11-C12-H12B 109.6 ; H12A-C12-H12B 108.1 ${ }^{\circ}$; C16-C15-H15 119.8 ; C16-C15-C14 120.4(4) ${ }^{\circ}$; C14-C15-H15 119.8 ; N2-C8-H8A 109.2 ${ }^{\circ}$; N2-C8-H8B

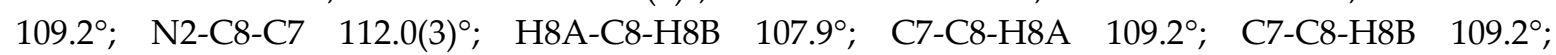
N2-C10-H10A $109.3^{\circ} ; \quad$ N2-C10-H10B $109.3^{\circ} ; \quad$ N2-C10-C9 111.8(3) ${ }^{\circ} ; \quad H 10 A-C 10-H 10 B \quad 107.9^{\circ}$; C9-C10-H10A 109.3; C9-C10-H10B 109.3; N1-C9-C10 112.5(3) ; N1-C9-H9A 109.1; N1-C9-H9B

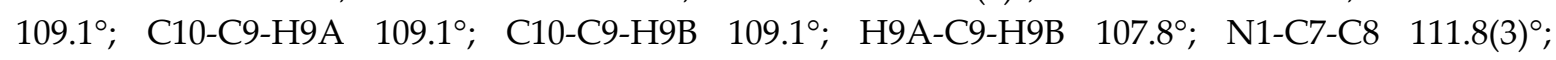

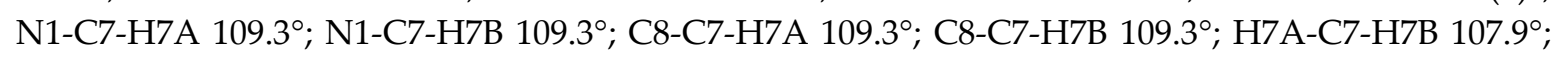
C13-C14-C15 121.9(4) ${ }^{\circ}$; C13-C14-H14 119.0 ; C15-C14-H14 119.0; C4-C5-H5 119.1; C4-C5-C6 121.9(5) ; C6-C5-H5 119.1 ${ }^{\circ}$; C6-C1-H1 121.3; C6-C1-C2 117.5(5) ${ }^{\circ}$; C2-C1-H1 121.3; C4-C3-H3 119.3;

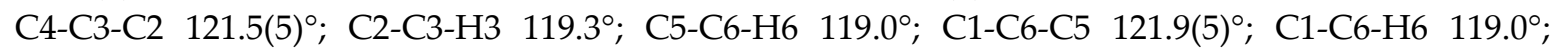
$\mathrm{C} 1-\mathrm{C} 2-\mathrm{C} 3121.6(5)^{\circ} ; \mathrm{C} 1-\mathrm{C} 2-\mathrm{H} 2119.2^{\circ} ; \mathrm{C} 3-\mathrm{C} 2-\mathrm{H} 2119.2^{\circ}$.

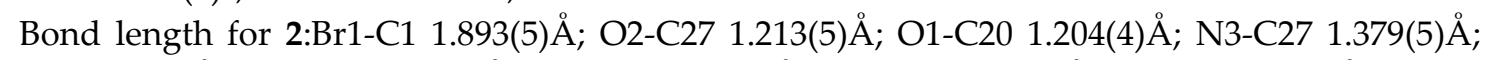

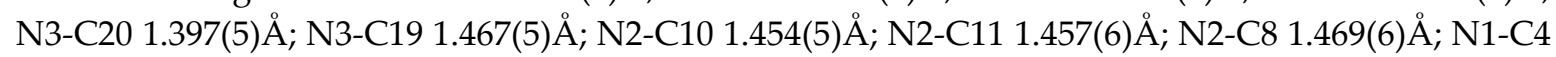

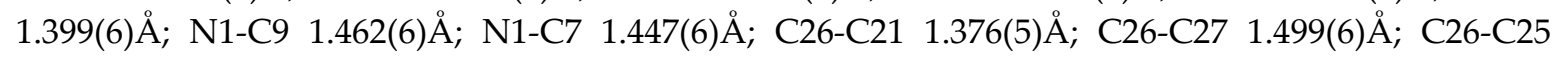
1.381(6)

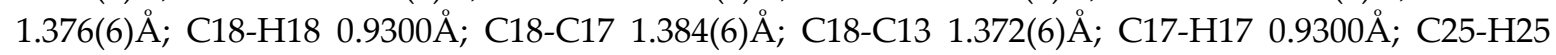

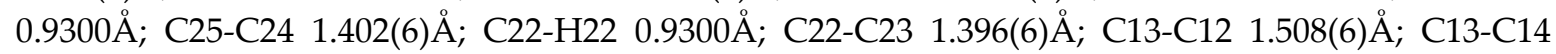

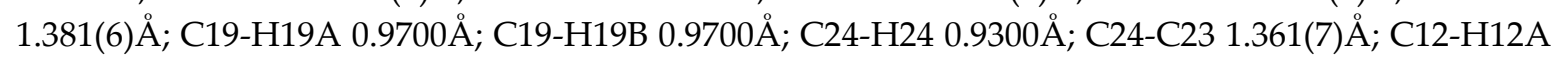

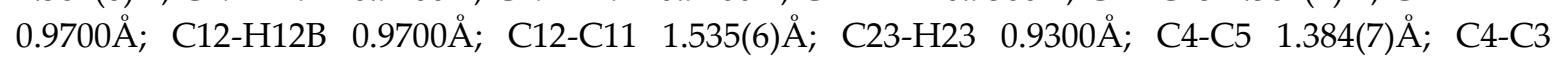

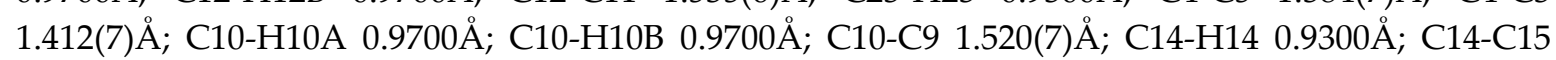

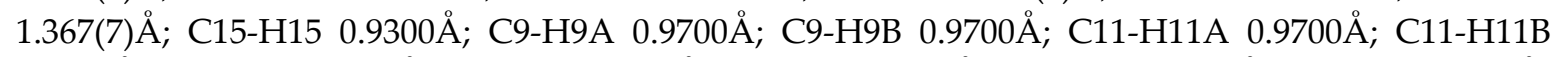

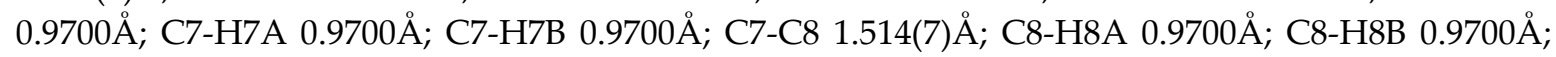

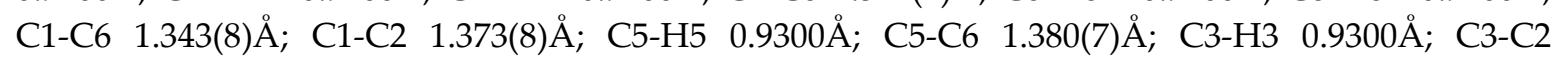

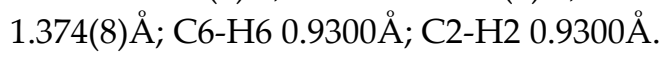

Bond angle for 2: C27-N3-C20 111.7(3) ${ }^{\circ}$; C27-N3-C19 124.1(3) ${ }^{\circ}$; C20-N3-C19 124.1(3) ${ }^{\circ}$; C10-N2-C11 113.3(4) ${ }^{\circ}$; C10-N2-C8 107.0(4) ${ }^{\circ}$; C11-N2-C8 108.6(4) ${ }^{\circ}$; C4-N1-C9 117.6(4) ${ }^{\circ}$; C4-N1-C7 116.1(4) $)^{\circ}$; C7-N1-C9 110.5(4) ${ }^{\circ}$; C21-C26-C27 107.6(4) ${ }^{\circ}$; C21-C26-C25 122.1(4) ${ }^{\circ}$; C25-C26-C27 130.2(4) ${ }^{\circ}$;

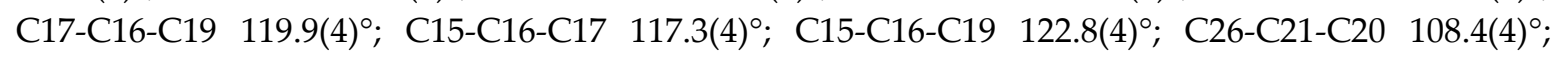

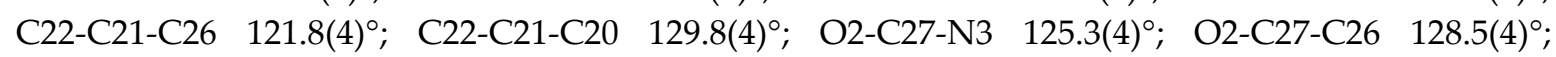
N3-C27-C26 106.2(3) ${ }^{\circ}$; C17-C18-H18 119.1 ${ }^{\circ}$; C13-C18-H18 119.1; C13-C18-C17 121.7(4) ${ }^{\circ}$; O1-C20-N3 124.4(4) ${ }^{\circ}$; O1-C20-C21 129.6(4) ${ }^{\circ}$; N3-C20-C21 106.1(3) ${ }^{\circ}$; C16-C17-H17 119.8 ; C18-C17-C16 120.3(4) ${ }^{\circ}$; 
C18-C17-H17 119.8 ${ }^{\circ} ; \quad$ C26-C25-H25 121.9 ; C26-C25-C24 116.2(4) ; C24-C25-H25 121.9 ; C21-C22-H22 121.8 ; C21-C22-C23 116.3(4) ; C23-C22-H22 121.8 ; C18-C13-C12 120.0(4) ${ }^{\circ}$; C18-C13-C14 117.7(4) ; C14-C13-C12 122.1(4) ; N3-C19-C16 112.5(3) ${ }^{\circ}$; N3-C19-H19A 109.1 ; N3-C19-H19B 109.1 ${ }^{\circ}$; C16-C19-H19A $109.1^{\circ}$; C16-C19-H19B 109.1 ${ }^{\circ}$; H19A-C19-H19B $107.8^{\circ}$;

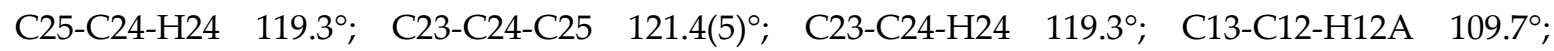

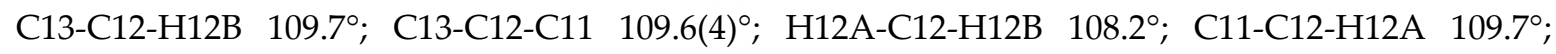

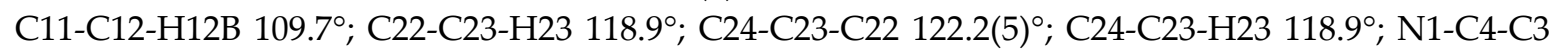
121.0(5) ; C5-C4-N1 123.4(5) ${ }^{\circ}$; C5-C4-C3 115.5(5) ${ }^{\circ}$; N2-C10-H10A 109.1; N2-C10-H10B 109.1 ; N2-C10-C9 112.6(4) ; H10A-C10-H10B 107.8 ; C9-C10-H10A 109.1 ; C9-C10-H10B 109.1 ; C13-C14-H14 119.4; C15-C14-C13 121.3(4) ; C15-C14-H14 119.4; C16-C15-H15 119.1; C14-C15-C16

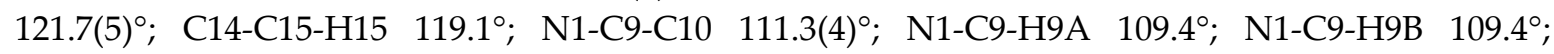
C10-C9-H9A $109.4^{\circ}$; C10-C9-H9B 109.4; H9A-C9-H9B 108.0 ; N2-C11-C12 115.3(4) ; N2-C11-H11A

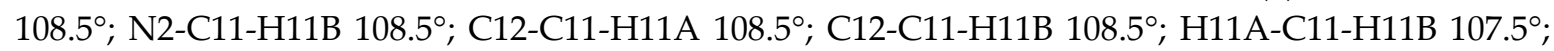
N1-C7-H7A 109.6 ; N1-C7-H7B 109.6 ; N1-C7-C8 110.2(4) ${ }^{\circ}$; H7A-C7-H7B 108.1 ${ }^{\circ}$; C8-C7-H7A 109.6 ${ }^{\circ}$;

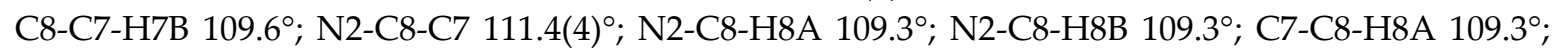
C7-C8-H8B 109.3; H8A-C8-H8B 108.0 ; C6-C1-Br1 121.5(5) ; C6-C1-C2 119.3(5) ; C2-C1-Br1

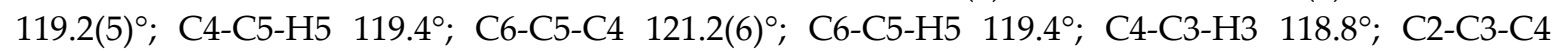
122.3(6) ; C2-C3-H3 118.8 ; C1-C6-C5 122.0(6) ; C1-C6-H6 119.0 ; C5-C6-H6 119.0 ; C1-C2-C3 $119.7(6)^{\circ} ; \mathrm{C} 1-\mathrm{C} 2-\mathrm{H} 2120.2^{\circ} ; \mathrm{C} 3-\mathrm{C} 2-\mathrm{H} 2120.2^{\circ}$.

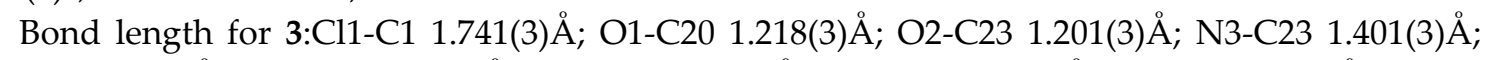

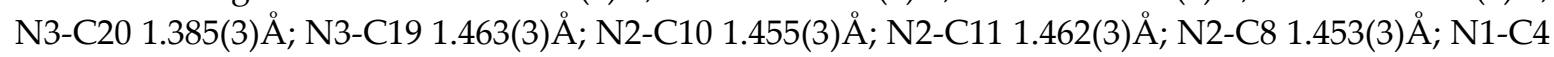
1.403(3) $\AA$; N1-C9 1.458(3)

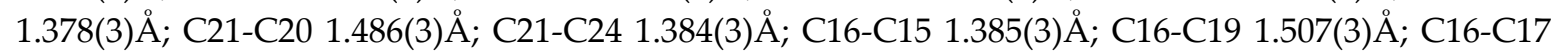

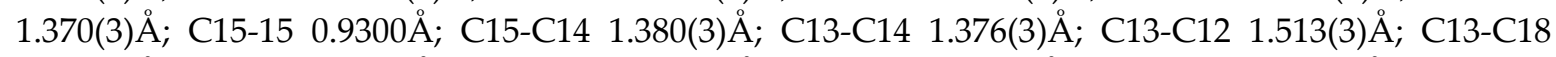

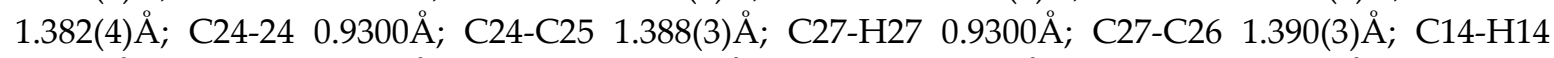

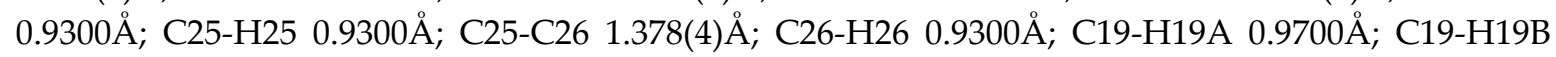

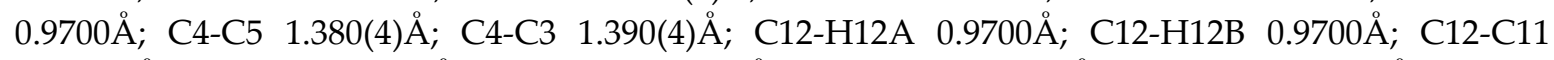

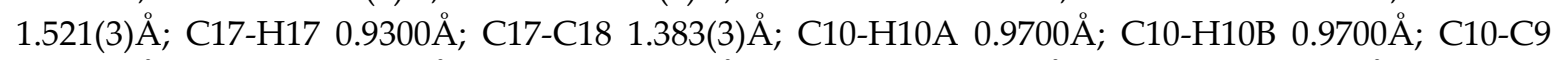

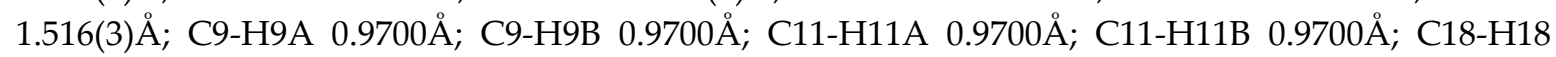

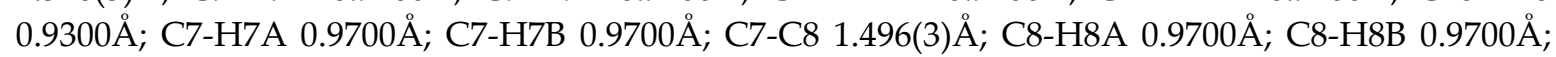

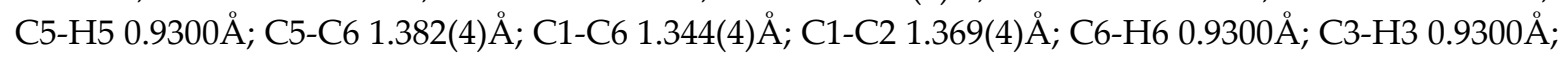
C3-C2 1.382(4) $\mathrm{A} ; \mathrm{C} 2-\mathrm{H} 20.9300 \AA$.

Bond angle for 3: C23-N3-C19 123.1(2) ${ }^{\circ}$; C20-N3-C23 111.78(18) ${ }^{\circ}$; C20-N3-C19 124.97(19) ${ }^{\circ}$; C10-N2-C11 113.1(2) ; C10-N2-C8 108.16(19) ; C8-N2-C11 109.17(19) ; C4-N1-C9 118.2(2) ; C4-N1-C7 116.3(2) ${ }^{\circ}$ C C7-N1-C9 110.24(19) ${ }^{\circ}$; C21-C22-C23 108.76(19) ; C27-C22-C21 121.8(2) ; C27-C22-C23 129.4(2) ; C22-C21-C20 107.59(19) ${ }^{\circ}$; C22-C21-C24 121.6(2) ; C24-C21-C20 130.8(2) ; O2-C23-N3 124.5(2); O2-C23-C22 130.1(2) ; N3-C23-C22 105.34(19) ; O1-C20-N3 124.8(2) ; O1-C20-C21 128.7(2) ${ }^{\circ}$; N3-C20-C21 106.51(19) ${ }^{\circ}$; C15-C16-C19 120.2(2) ${ }^{\circ}$; C17-C16-C15 117.5(2) C17-C16-C19 122.3(2) ; C16-C15-H15 119.3; C14-C15-C16 121.4(2); C14-C15-H15 119.3; C14-C13-C12 120.4(2) ; C14-C13-C18 117.2(2) ; C18-C13-C12 122.2(2) ${ }^{\circ}$; C21-C24-H24 121.6 ; C21-C24-C25 116.8(2) ; C25-C24-H24 121.6 ; C22-C27-H27 121.6 ; C22-C27-C26 116.7(2) ; C26-C27-H27 121.6 ; C15-C14-H14 119.4 ; C13-C14-C15 121.2(2) ; C13-C14-H14 119.4 ;

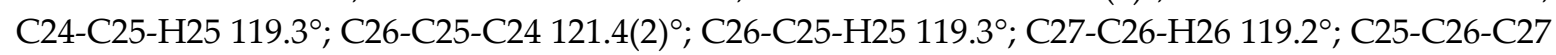
121.6(2) ; C25-C26-H26 119.2 ; N3-C19-C16 112.93(18) ; N3-C19-H19A 109.0 ; N3-C19-H19B 109.0 ; C16-C19-H19A 109.0 ; C16-C19-H19B 109.0 ; H19A-C19-H19B 107.8 ; C5-C4-N1 122.8(3) ; C5-C4-C3 116.0(2) ; C3-C4-N1 121.2(2) ; C13-C12-H12A 109.8 ; C13-C12-H12B 109.8 ; C13-C12-C11 109.5(2) ; H12A-C12-H12B 108.2 ; C11-C12-H12A 109.8 ; C11-C12-H12B 109.8 ; C16-C17-H17 119.5 ; C16-C17-C18 121.0(2) ; C18-C17-H17 119.5 ; N2-C10-H10A 109.2 ; N2-C10-H10B 109.2 ${ }^{\circ}$; N2-C10-C9

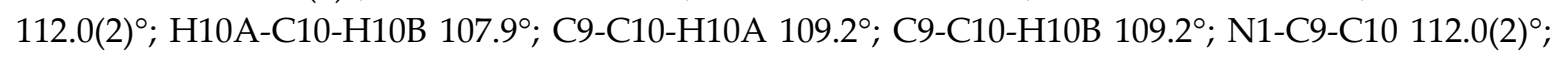
N1-C9-H9A $109.2^{\circ}$; N1-C9-H9B 109.2 ${ }^{\circ}$; C10-C9-H9A 109.2 ; C10-C9-H9B 109.2 ; H9A-C9-H9B 107.9 ; N2-C11-C12 115.1(2); N2-C11-H11A 108.5 ; N2-C11-H11B 108.5 ; C12-C11-H11A 108.5 ; C12-C11-H11B 108.5 ; H11A-C11-H11B 107.5 ; C13-C18-C17 121.7(2); C13-C18-H18 119.2 ; 
C17-C18-H18 119.2 ${ }^{\circ}$; N1-C7-H7A 109.4 ${ }^{\circ}$ N1-C7-H7B 109.4 ; N1-C7-C8 111.0(2) ; H7A-C7-H7B 108.0 ; C8-C7-H7A 109.4 ${ }^{\circ}$; C8-C7-H7B 109.4 ; N2-C8-C7 112.2(2) ${ }^{\circ}$; N2-C8-H8A 109.2 ${ }^{\circ}$; N2-C8-H8B

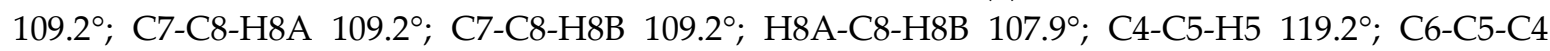
121.6(3) ${ }^{\circ}$; C6-C5-H5 119.2 ${ }^{\circ}$; C6-C1-Cl1 120.7(3) ${ }^{\circ}$; C6-C1-C2 119.5(3) ${ }^{\circ}$; C2-C1-Cl1 119.9(3) ${ }^{\circ}$ C5-C6-H6

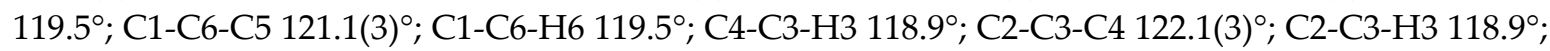
C1-C2-C3 119.7(3) ${ }^{\circ}$; C1-C2-H2 120.2 ${ }^{\circ}$; C3-C2-H2 120.2 ${ }^{\circ}$.

All these compounds expanded along a axis through pi-pi interaction shown in Fig 3 . All these compounds expanded into 3D-network via the intermolecular weak hydrogen bonds. Compound 2 and 3 showed halogen interactions between molecules nearby different from compound 1, which resulted in the large difference of cell parameter of compound 1 (Figure 4). In summary, the dihedral angle values, the bond lengths and the parameters demonstrated that involvement of halogen atoms did affect the conformation and the packing mode of compounds.

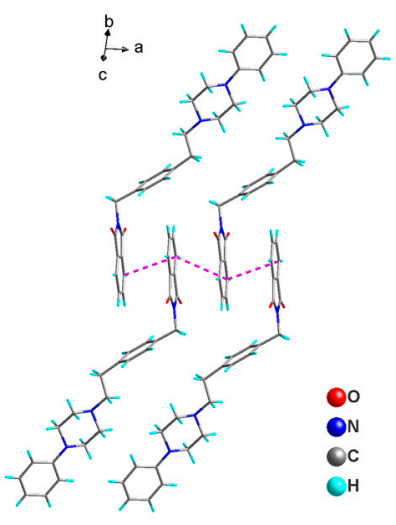

(a)

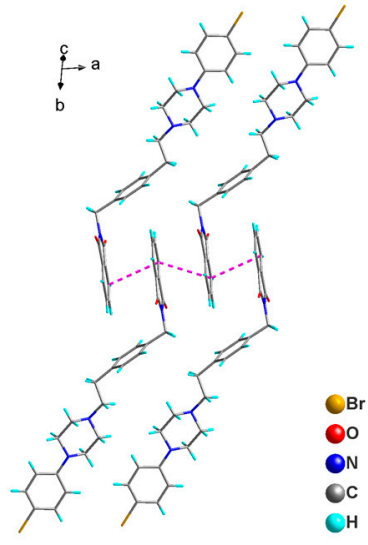

(b)

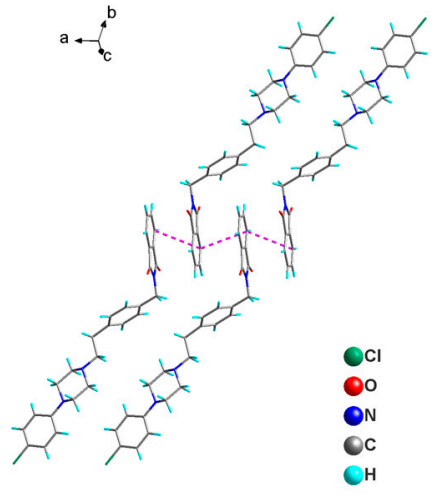

(c)

Figure 3. Pi-pi interactions of compound 1(a), compound 2(b), compound 3(c).Purple dashed lines show the pi-pi interactions.

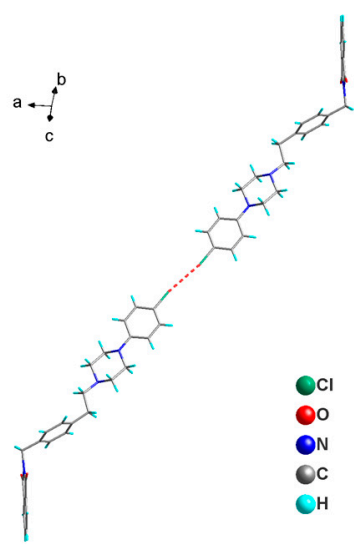

(a)

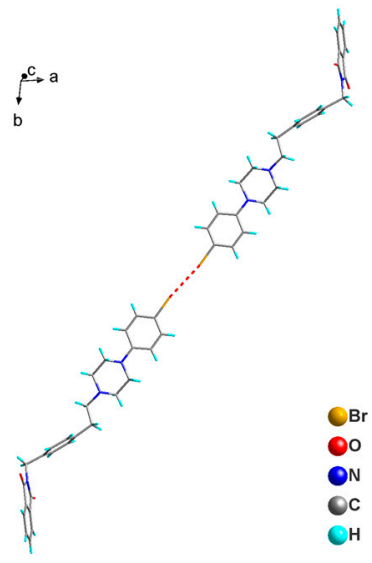

(b)

Figure 4. Halogen interactions of compound 2(a), compound 3(b). Red dashed lines show the halogen interactions.

\section{Experimental Section}

\subsection{Materials and Instrumentation}

NMR spectra were recorded on a Brucker ADVANCE 400MHz spectrometer (Bruker, Billerica, MA, USA) and chemical shifts of the $1 \mathrm{H}$, and $13 \mathrm{C}$ spectra were reported in parts per million (ppm) 
using the solvent shifts for $1 \mathrm{H}$ and $13 \mathrm{C}$ as internal standard $\left(\mathrm{CDCl}_{3}: 1 \mathrm{H} \mathrm{d}=7.26,13 \mathrm{C} \mathrm{d}=77.0\right)$. MS spectra were determined by electron spray ionization using an Electron Finnigan MAT 95-XP mass spectrometer (Thermo Fisher Scientific, Waltham, MA, USA). X-ray single crystal experiments were performed on a Bruker P4 diffractometer (Bruker, Billerica, MA, USA).

\subsection{Preparation of Three Compounds}

General procedure for the synthesis of piperazinyl isoindoline-1,3-diones. To a mixture of 2-(4-((1,3-Dioxoisoindolin-2-yl)methyl)phenyl)ethyl-4-methylbenzenesulfonate(217.8mg,0.5mmol) in $50 \mathrm{~mL}$ EtOH and $\mathrm{KOH}(112.2 \mathrm{mg}, 2.0 \mathrm{mmol})$, Arylpiperazines $(0.55 \mathrm{mmol})$ was added at ambient temperature. The reaction mixture was stirred and heated to reflux for 2-3h, until TLC indicated the end of reaction. Solvent was removed under reduced pressure, and the residue was extracted with $\mathrm{CH}_{2} \mathrm{Cl}_{2}(3 \times 100 \mathrm{~mL})$. The combined organic layer was successively washed with water, brine, dried over anhydrous sodium sulfate, and concentrated in vacuo. The residue was purified by silica gel column chromatography using ethyl acetate/petroleum ether $(1: 8, \mathrm{v} / \mathrm{v})$ as eluent, to afford Piperazinyl isoindoline-1,3-diones as white solid.

2-(4-(2-(4-Phenylpiperazin-1-yl)ethyl)benzyl)isoindoline-1,3-dione (1). White solid, mp.128-129, $1 \mathrm{H}$ NMR $\left(400 \mathrm{MHz}, \mathrm{CDCl}_{3}\right) \mathrm{d}$ in ppm: $7.838(\mathrm{dd}, \mathrm{J}=5.4,3.0 \mathrm{~Hz}, 2 \mathrm{H}), 7.700(\mathrm{dd}, \mathrm{J}=5.4,3.0 \mathrm{~Hz}, 2 \mathrm{H}), 7.368$ $(\mathrm{d}, \mathrm{J}=8.0 \mathrm{~Hz}, 2 \mathrm{H}), 7.281-7.242(\mathrm{~m}, 2 \mathrm{H}), 7.178(\mathrm{~d}, \mathrm{~J}=8.0 \mathrm{~Hz}, 2 \mathrm{H}), 6.927(\mathrm{~d}, \mathrm{~J}=8.0 \mathrm{~Hz}, 2 \mathrm{H}), 6.860(\mathrm{t}, \mathrm{J}=$ $7.3 \mathrm{~Hz}, 1 \mathrm{H}), 4.820(\mathrm{~s}, 2 \mathrm{H}), 3.240(\mathrm{~m}, 4 \mathrm{H}), 2.830(\mathrm{~m}, 2 \mathrm{H}), 2.680(\mathrm{~m}, 6 \mathrm{H}) ; 13 \mathrm{CNMR}\left(101 \mathrm{MHz}, \mathrm{CDCl}_{3}\right) \mathrm{d}$ in ppm: $168.05,151.31,139.90,134.23,133.96,132.17,129.11,129.03,128.79,123.32,119.71,116.07$, 77.37,77.05,76.73,60.35, 53.22, 49.14, 41.34, 33.25; HRMS (ESI) m/z [M+1]+: calcd for $\mathrm{C}_{27} \mathrm{H}_{28} \mathrm{O}_{2} \mathrm{~N}_{3}$, 426.2176, found, 426.2166 .

2-(4-(2-(4-(4-Bromophenyl)piperazin-1-yl)ethyl)benzyl)isoindoline-1,3-dione (2). White solid, mp.173-174 ${ }^{\circ}, 1 \mathrm{HNMR}\left(400 \mathrm{MHz}, \mathrm{CDCl}_{3}\right) \mathrm{d}$ in ppm: $7.838(\mathrm{dd}, \mathrm{J}=5.4,3.0 \mathrm{~Hz}, 2 \mathrm{H}), 7.701(\mathrm{dd}, \mathrm{J}=5.4,3.0$ $\mathrm{Hz}, 2 \mathrm{H}), 7.351(\mathrm{dd}, \mathrm{J}=12.0,3.0 \mathrm{~Hz}, 2 \mathrm{H}), 7.259(\mathrm{~s}, 2 \mathrm{H}), 7.172(\mathrm{~d}, \mathrm{~J}=8.0 \mathrm{~Hz}, 2 \mathrm{H}), 6.780(\mathrm{dd}, \mathrm{J}=6.8,2.0 \mathrm{~Hz}$, 2H), $4.819(\mathrm{~s}, 2 \mathrm{H}), 3.211(\mathrm{~s}, 4 \mathrm{H}), 2.830(\mathrm{~s}, 2 \mathrm{H}), 2.669(\mathrm{~s}, 6 \mathrm{H}) ; 13 \mathrm{C} \mathrm{NMR}\left(101 \mathrm{MHz}, \mathrm{CDCl}_{3}\right) \mathrm{d}$ in ppm: $168.04,150.31,139.32,134.25,133.97,132.16,131.85,129.01,128.78,123.32,117.60$, 111.77,77.37,77.06,76.74, 60.25, 53.01, 48.97, 41.33, 33.23; HRMS (ESI) m/z [M+1]+: calcd for $\mathrm{C}_{27} \mathrm{H}_{27} \mathrm{O}_{2} \mathrm{~N}_{3} \mathrm{Br}$, 504.1281, found, 504.1269.

2-(4-(2-(4-(4-Chlorophenyl)piperazin-1-yl)ethyl)benzyl)isoindoline-1,3-dione (3). White solid, mp.167-168 ${ }^{\circ}, 1 \mathrm{HNMR}\left(400 \mathrm{MHz}, \mathrm{CDCl}_{3}\right) \mathrm{d}$ in ppm: $7.830(\mathrm{dd}, \mathrm{J}=6.5,3.2 \mathrm{~Hz}, 2 \mathrm{H}), 7.708(\mathrm{dd}, \mathrm{J}=6.5,3.2$ $\mathrm{Hz}, 2 \mathrm{H}), 7.363(\mathrm{~d}, \mathrm{~J}=8.0 \mathrm{~Hz}, 2 \mathrm{H}), 7.209(\mathrm{dd}, \mathrm{J}=7.2,2.4 \mathrm{~Hz}, 2 \mathrm{H}), 7.170(\mathrm{~d}, \mathrm{~J}=8.0 \mathrm{~Hz}, 2 \mathrm{H}), 6.831(\mathrm{dd}, \mathrm{J}=$ 7.2, $2.4 \mathrm{~Hz}, 2 \mathrm{H}), 4.818(\mathrm{~s}, 2 \mathrm{H}), 3.184(\mathrm{~s}, 4 \mathrm{H}), 2.809(\mathrm{~s}, 2 \mathrm{H}), 2.655(\mathrm{~s}, 6 \mathrm{H}) ; 13 \mathrm{CNMR}\left(101 \mathrm{MHz}, \mathrm{CDCl}_{3}\right) \mathrm{d}$ in ppm: 168.04, 149.90, 139.81, 134.25, 133.97, 132.15, 129.01, 128.94, 128.78,124.52, 123.32, 117.22,77.36,77.04,76.73,60.24, 53.03, 49.13, 41.33, 33.21; HRMS (ESI) $\mathrm{m} / \mathrm{z}[\mathrm{M}+1]+$ : calcd for $\mathrm{C}_{27} \mathrm{H}_{27} \mathrm{O}_{2} \mathrm{~N}_{3} \mathrm{Cl}, 460.1786$, found, 460.1779 .

\subsection{Crystal Structure Determination}

Crystals suitable for diffraction experiment were collected from solutions. The data were collected by Crystal data, data collection and structure refinement details are summarized in Table 1 . The structure was solved and refined using the OLEX2 program suite [17], equipped with ShelXT and ShelXL program [18]. All non-hydrogen atoms were located from Fourier map directly by ShelXT and refined anisotropically. Hydrogen atoms on all non-hydrogen atoms were placed in calculated positions, and their coordinates and displacement parameters were constrained to ride on the carrier atoms. All the CIF files could be downloaded from http://www.ccdc.cam.ac.uk.

Table 1.Crystal data for 1-3

(1)

Crystal data

Chemical formula $\mathrm{Mr}$

Crystal system, space group
(2)

(3)
$\mathrm{C}_{27} \mathrm{H}_{26} \mathrm{ClN}_{3} \mathrm{O}_{2}$ 459.96

Triclinic, P-1
504.42
$\mathrm{H}_{26} \mathrm{BrN}_{3} \mathrm{O}$

Triclinic, P-1
Triclinic, P-1 


\begin{tabular}{|c|c|c|c|}
\hline Temperature (K) & 293 & 293 & 293 \\
\hline$a, b, c(\AA)$ & $\begin{array}{c}6.9159(14), 9.999(2), \\
16.925(3)\end{array}$ & $\begin{array}{c}6.9995(14), 9.919(2), \\
17.671(4)\end{array}$ & $\begin{array}{c}6.9872(14), 9.863(2) \\
17.557(4)\end{array}$ \\
\hline$\alpha, \beta, \gamma\left({ }^{\circ}\right)$ & $\begin{array}{c}88.25(3), 85.14(3) \\
79.22(3)\end{array}$ & $\begin{array}{c}97.55(3), 92.19(3) \\
102.23(3)\end{array}$ & $\begin{array}{c}96.81(3), 91.89(3) \\
101.74(3)\end{array}$ \\
\hline$V\left(\AA^{3}\right)$ & $1145.5(4)$ & $1185.9(4)$ & $1174.2(4)$ \\
\hline Z & 2 & 2 & 2 \\
\hline Radiation type & $\mathrm{Cu} \mathrm{K} \alpha$ & $\mathrm{Cu} \mathrm{K} \alpha$ & $\mathrm{Cu} \mathrm{K} \alpha$ \\
\hline$\mu(\mathrm{mm}-1)$ & 0.62 & 2.59 & 1.67 \\
\hline Crystal size $(\mathrm{mm})$ & $0.3 \times 0.2 \times 0.2$ & $0.3 \times 0.2 \times 0.2$ & $0.3 \times 0.2 \times 0.2$ \\
\hline \multicolumn{4}{|l|}{ Data collection } \\
\hline Diffractometer & $\begin{array}{c}\text { Bruker P4 } \\
\text { diffractometer }\end{array}$ & $\begin{array}{c}\text { Bruker P4 } \\
\text { diffractometer }\end{array}$ & $\begin{array}{c}\text { Bruker P4 } \\
\text { diffractometer }\end{array}$ \\
\hline Absorption correction & None & None & None \\
\hline $\begin{array}{c}\text { No. of measured, } \\
\text { independent and } \\
\text { observed }[\mathrm{I}>2 \sigma(\mathrm{I})] \\
\text { reflections }\end{array}$ & $12809,4084,1981$ & $13126,4224,2491$ & $13592,4219,2776$ \\
\hline Rint & 0.056 & 0.164 & 0.103 \\
\hline$(\sin \theta / \lambda) \max \left(\AA^{-1}\right)$ & 0.602 & 0.602 & 0.602 \\
\hline \multicolumn{4}{|l|}{ Refinement } \\
\hline $\mathrm{R}\left[F^{2}>2 \sigma\left(F^{2}\right)\right], \mathrm{wR}\left(F^{2}\right), \mathrm{S}$ & $0.074,0.282,1.10$ & $0.079,0.249,1.08$ & $0.049,0.152,1.01$ \\
\hline No. of reflections & 4084 & 4224 & 4219 \\
\hline No. of parameters & 290 & 299 & 299 \\
\hline No. of restraints & 0 & 0 & 0 \\
\hline H-atom treatment & $\begin{array}{l}\text { H-atom parameters } \\
\text { constrained }\end{array}$ & $\begin{array}{l}\text { H-atom parameters } \\
\text { constrained }\end{array}$ & $\begin{array}{l}\text { H-atom parameters } \\
\text { constrained }\end{array}$ \\
\hline$\Delta$ @max, $\Delta$ @min $\left(\mathrm{e} \AA^{-3}\right)$ & $0.24,-0.20$ & $0.66,-0.79$ & $0.17,-0.23$ \\
\hline CCDC Number & 1445031 & 1445032 & 1445033 \\
\hline
\end{tabular}

Computer programs: ShelXT (Sheldrick, 2015), SHELXL (Sheldrick, 2015), Olex2 (Dolomanov et al., 2009).

\section{Conclusions}

Three

new

arylpiperazine

derivatives,

2-(4-(2-(4-Phenylpiperazin-1-yl)ethyl)benzyl)isoindoline-1,3-dione

2-(4-(2-(4-(4-Bromophenyl)piperazin-1-yl)ethyl)benzyl)isoindoline-1,3-dione

(2) $(1)$,

2-(4-(2-(4-(4-Chlorophenyl)piperazin-1-yl)ethyl)benzyl)isoindoline-1,3-dione (3) were synthesized via classical synthesis method.Their structures were determined via NMR, MS and X-ray single crystal diffraction analysis. Pi-pi interactions were observed between molecules, and compound 2 and 3 showed halogen interactions between molecules nearby, which was different from compound1.The dihedral angle values, the bond lengths and the parameters demonstrated that involvement of halogen atoms did affect the conformation and the packing mode of the compounds.

Acknowledgments: This project was supported by the Natural Science Foundation of Shandong Province, China (No. ZR2011HL003).

Author Contributions: Jinhui Zhou and Guoqiang Shangguan conceived and designed the experiments;Jinhui Zhou,Maocai Yan and Maojian Shiperformed the experiments; Jun Xu and Guoqiang Shangguan analyzed the data; Jinhui Zhouwrote the paper.

Conflicts of Interest: The authors declare no conflict of interest.

\section{References}

1. Shah, S.A.; Rivera, G.; Ashfaq, M. Recent Advances in Medicinal Chemistry of Sulfonamides. Rational 
Design as Anti-Tumoral, Anti-Bacterial and Anti-Inflammatory Agents. Mini-Rev.Med.Chem. 2013, 13, 70-86. [CrossRef]

2. Wang, J.H.; Wang, Q.D.; Dun, Y.Y.; Fang, H. Syntheses and Antitumor Activities of Purine sulfonamides Derivatives. Chem. J. Chinese Universities 2014, 35, 1189-1198. [CrossRef]

3. Fang, Xu; Hong, Chen; Jingyi, Xu; Bing-Bing, Xu; Bin-Hao, Shao; Bi-Yun, Huang; Mu, Yuan Design, synthesis and biological evaluation of novel arylpiperazine derivatives on human prostate cancer cell lines Chin. Chem. Lett. 2015, 27, 277-282.[CrossRef]

4. F. Berardi; C. Abate; S. Ferorelli; Robertis, A.F.; Leopoldo, M.; Colabufo, N.A. Novel 4-(4-Aryl)cyclohexyl-1-(2-pyridyl)piperazines as $\Delta_{8}-\Delta_{7}$ Sterol Isomerase (Emopamil Binding Protein) Selective Ligands with Antiproliferative Activity. J. Med. Chem. 2008, 51, 7523-7531.[CrossRef]

5. Abate, C.; Niso, M.; Contino, M.; Colabufo, N.A.; Feroselli, S. 1-Cyclohexyl-4-(4-arylcyclohexyl)piperazines: Mixed $\sigma$ and Human $\Delta_{8}-\Delta 7$ Sterol Isomerase Ligands with Antiproliferative and P-Glycoprotein Inhibitory Activity. ChemMedChem. 2011, 6, 73-80.[CrossRef]

6. W.H. Liu; J.X. Chang; Y. Liu; J.W. Luo. Design, synthesis and activities of novel benzothiazole derivatives containing arylpiperazine. Acta Pharmaceut. Sin. 2013, 48, 1259-1265. [CrossRef]

7. M. Dellabella; G. Milanese; G. Muzzonigro. Efficacy of Tamsulosin in the Medical Management of Juxtavesical Ureteral Stones. J. Urol. 2003, 170, 2202-2205. [CrossRef]

8. Y. Nishino; T. Masue; K. Miwa; Y. Takahashi; S. Ishihara; T. Deguchi Comparison of two $\alpha_{1}$-adrenoceptor antagonists, naftopidil and tamsulosin hydrochloride, in the treatment of lower urinary tract symptoms with benign prostatic hyperplasia: a randomized crossover study. BJU Int.2006, 97, 747-751. [CrossRef]

9. Y. Kojima; S. Sasaki; Y. Kubota; M. Hayase; Y. Hayashi; H. Shinoura; G. Tsujimoto; K. Kohri. Expression of $\alpha_{1}$-Adrenoceptor Subtype mRNA as a Predictor of the Efficacy of Subtype Selective $\alpha_{1}$-Adrenoceptor Antagonists in the Management of Benign Prostatic Hyperplasia. J. Urol. 2008, 179, 1040-1046. [CrossRef]

10. Y. Hori; K. Ishii; H. Kanda; Y. Iwamoto; K. Nishikawa; N. Soga; H. Kise; K. Arima; Y. Sugimura Naftopidil, a Selective $\alpha_{1}$-Adrenoceptor Antagonist, Suppresses Human Prostate Tumor Growth by Altering Interactions between Tumor Cells and Stroma. Cancer Prev. Res. 2011, 4, 87-96. [CrossRef]

11. H. Kanda; K. Ishii; Y. Ogura; T. Imamura; M. Kanai; K. Arima; Y. Sugimura Naftopidil, a selective $\alpha-1$ adrenoceptor antagonist, inhibits growth of human prostate cancer cells by G1 cell cycle arrest. Int. J. Cancer. 2008, 122, 444-451. [CrossRef]

12. E. Masachika; T. Kanno; T. Nakano; A. Gotoh. Naftopidil induces apoptosis in malignant mesothelioma cell lines independently of $\alpha 1$-adrenoceptor blocking. Anticancer Res. 2013, 33, 887-894.[PubMed]

13. Desiraju, G R; Ho, P S; Kloo, L.; A. C. Legon; R. Marquardt; P. Metrangolo; P. Politzer; G. Resnat; K. Rissanen. Definition of the halogen bond (IUPAC Recommendations 2013). Pure Appl. Chem. 2013, 85, 1711-1713.[CrossRef]

14. Aakeröy, C. B.; Fasulo, M.; Schultheiss, N.; Desper, J.; Moore, C. Structural competition between hydrogen bonds and halogen bonds. J. Am. Chem. Soc. 2007, 129, 13772-13773.[CrossRef]

15. Priimagi, A.; Cavallo, G.; Metrangolo P.; Resnati, G. The Halogen Bond in the Design of Functional Supramolecular Materials: Recent Advances. Acc. Chem. Res. 2013, 46, 2686-2695.[CrossRef]

16. Voth, A R; Hays, F A; Ho, P S. Directing macromolecular conformation through halogen bonds. Proc. Natl. Acad. Sci. U. S. A. 2007, 104, 6188-6193.[CrossRef]]. [PubMed]

17. Dolomanov, O. V.; Bourhis, L. J.; Gildea, R. J.; OLEX2: a complete structure solution, refinement and analysis program. J APPL CRYSTALLOGR. 2009, 42, 339-341.[CrossRef]

18. Sheldrick, G. M. Crystal structure refinement with SHELXL. Acta Crystallogr., Sect. C: Struct. Chem. 2015, 
9 of 9

71, 3-8.[CrossRef]

(C) 2016 by the authors; licensee Preprints, Basel, Switzerland. This article is an open access article distributed under the terms and conditions of the Creative Commons by Attribution (CC-BY) license (http://creativecommons.org/licenses/by/4.0/). 